EOMmunutriln? Communication et organisation

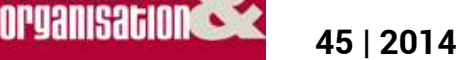

Risques mineurs, changements majeurs

\title{
Risques organisationnels et anticipation
}

Une communication complexifiée autour du near-miss

Organizational risks and anticipation. A complexified communication around the near-miss

Sylvie P. Alemanno

(2) OpenEdition

Journals

Édition électronique

URL : http://journals.openedition.org/communicationorganisation/4494

DOI : 10.4000/communicationorganisation.4494

ISSN : 1775-3546

Éditeur

Presses universitaires de Bordeaux

Édition imprimée

Date de publication : 1 juin 2014

Pagination : $59-72$

ISBN : 978-2-86781-904-9

ISSN : $1168-5549$

Référence électronique

Sylvie P. Alemanno, «Risques organisationnels et anticipation », Communication et organisation [En ligne], 45 | 2014, mis en ligne le 01 juin 2017, consulté le 30 avril 2019. URL : http:// journals.openedition.org/communicationorganisation/4494; DOI : 10.4000/

communicationorganisation.4494 


\title{
Risques organisationnels et anticipation une communication complexifiée autour du near-miss
}

\author{
Sụlvie P. Alemanno ${ }^{1}$
}

\section{Introduction}

L'existence du risque dans et par les organisations s'associe toujours à sa gestion, son évaluation et sa prévention, à son anticipation dans la collecte des alertes. Quel que soit le type de risque, il est corrélé (en général) à une mesure du danger qui va de la contingence faible (risques mineurs) à la probabilité forte à conséquences vitales (risques majeurs). Si dans tout type d'organisation la neutralisation des risques est une préoccupation centrale, c'est dans le domaine de la santé (notamment dans les pays occidentaux) qu'elle est devenue particulièrement aigüe.

Les organisations de santé, publiques et privées, assurent une veille attentive intra-muros, veille régie par des normes légales (article 51 Loi HPST)2 ${ }^{2}$ Les processus d'information et de communication occupent une place de plus en plus importante dans la mécanique de gestion des risques. Selon l'approche fonctionnaliste des sciences de l'administration et du management de la santé, transmettre les informations suffit à l'application de normes procédurales édictées par l'État. Ce point de vue se retrouve dans les pratiques managériales des directeurs d'établissements. La prescription de vigilance concernant les risques, qui se fait dans le sens up-down, est proposée-imposée aux équipes de terrain. «Elle repose sur une démarche méthodique d'identification des risques a priori et de leur niveau de gravité pour chaque secteur de l'établissement, sur l'identification a posteriori à partir des événements indésirables, des erreurs, des situations à risque, des incidents, accidents survenant au sein de l'organisation, et sur l'identification des crises prévisibles. » (FHP, 2011). En termes de gestion, cette démarche méthodique renvoie au travail des diverses

1 Sylvie P. Alemanno est enseignante-chercheure en Sciences de l'Information et de la Communication (SIC) au Laboratoire Information, Milieu, Médiations (I3M, EA 3820) de l'Université de Nice Sophia Antipolis ; Sylvie.Parrini-Alemanno@unice.fr

2 Article 51 de la loi Hôpital, Patients, Santé, Territoires (Cf. document Haute Autorité de Santé : « La sécurité des patients. Mettre en œuvre la gestion des risques associés aux soins en établissements de santé. Des concepts à la pratique. » (HAS, 2012). 
commissions de vigilance qui tentent de récupérer des informations pour organiser leurs actions.

Les études en communication organisationnelle dans le champ des sciences de l'information et de la communication, nous ont semblé plus pertinentes pour comprendre les processus à l'œuvre dans la dynamique de vigilance collective des risques. Nous abordons les organisations de santé du point de vue de la théorie des systèmes sociaux pour saisir le changement dans la complexité, et nous la combinons avec la théorie de construction du sens pour comprendre la collectivisation des actions. Nous montrons dans cet article qu'une action distribuée et partagée aux différents niveaux professionnels, génère un espace d'échanges dynamiques de traitement de l'information, propice non seulement à l'identification de risques professionnels mais aussi à la mise en place d'un dispositif en quelque sorte en amont de la prévention. Nous étudions la mise en place dans un établissement de santé d'un dispositif de vigilance spéciale au moindre événement susceptible d'entraîner un accident, ce que l'on appelle near-miss ou "presqu'accident " et qui est un cas particulier d'événements indésirables $(\mathrm{EI})^{3}$. La procédure choisie implique chacun des professionnels de santé de l'établissement et va au-delà des normes officielles appliquées dans la plupart des établissements médicaux. À partir de ce dispositif de near-miss, nous examinons la tension entre une politique d'établissement d'apparence ultra sécuritaire, susceptible d'alourdir encore l'application des normes techniques de sécurité (par la traque systématique du moindre indice de danger), et l'instauration d'une nouvelle forme d'implication de tous les personnels quel que soit leur niveau hiérarchique.

L'apport des études en communication organisationnelle consiste, dans ce cas, à avoir plus de prise, d'une part sur la dynamique communicationnelle singulière autour du near-miss, d'autre part sur la manière dont cette dernière génère un espace où les acteurs affranchis des pressions hiérarchiques, s'engagent dans un processus relationnel fondé sur la confiance au management (Luhmann, 1994-2010). En effet, dans l'environnement des organisations de santé où les métiers sont pour la plupart à pratiques prudentielles, la notion de risque accompagne les professionnels dans chacune de leurs activités. Elle ne se départit pas de celle de confiance en eux-mêmes, en la direction qui les conduit et qui installe les conditions d'émergence de nouvelles pratiques professionnelles instaurées et renforcées par des formes de communications spécifiques.

Pour étudier ce phénomène d'une construction communicationnelle originale autour du near-miss, nous présenterons des données de contexte

3 «Un événement indésirable est un événement défavorable survenant chez un patient ou dans le processus de soin, quelles qu'en soient la gravité ou la nature, consécutif aux stratégies et actes de prévention, de diagnostic, de traitement, de soins, ou de réhabilitation. Il s'agit d'un événement qui s'écarte des résultats escomptés ou des attentes du soin et qui n'est pas lié à l'évolution naturelle de la maladie » in Haute Autorité de Santé, Gestion des risques et protocoles de coopération (Article 51 Loi HPST) Document d'aide pour les professionnels de santé, février 2012, p. 9. 
général sur les risques, les normes qui structurent les organisations, les épistémé que nous mobilisons, et le dispositif de near-miss. Notre démarche compréhensive de chercheur impliqué dans le suivi de cette procédure, nous permet de décrire à partir de ce focus sur le near-miss, les tensions induites par une politique d'établissement dans l'application de procédures nouvelles, leur appropriation individuelle et collective et le processus de confiance qui peut en découler.

\section{Une communication organisante dans l'environnement des systèmes sociaux : éléments de théories}

Dans la perspective de l'interactionnisme symbolique, nous considérons l'organisation comme une trame d'interactions multiples et mouvantes, caractérisée par la construction du sens que les acteurs, co-auteurs, donnent à leurs actes. Les individus sont définis par leurs comportements verbaux et non verbaux, et leurs actions dans les situations où ils sont en relation et auxquelles ils donnent un sens partagé, " chacun s'accordant sur l'autre » (Winkin, $2000: 7$ ). Bateson (1972) précise l'interdépendance attachée aux interactions des individus entre eux, avec le système et avec l'environnement dans une inter-construction croisée, constante et en équilibre instable. Ce point de vue constructiviste est radicalisé par K. Weick (1995) qui exprime avec l'ongoing, un processus continu d'élaboration de sens (sensemaking et sensegiving) dans et par la communication qui construit ainsi l'organisation (organizing). Lorganisation est envisagée comme un environnement créé, car interprété par les individus à travers leurs expériences. Et le sens collectif qui en résulte est déterminant pour la coordination des actions. Si le rôle du langage est fondamental dans l'approche de l'action stratégique de Weick, nous pensons toutefois que le processus communicationnel met en jeu d'autres composants non verbaux, techniques, environnementaux réalisant des formes d'organisations complexes, métastables.

Le processus d'attribution et de partage du sens des actions prend une importance déterminante dans les environnements complexes et en mutation comme les organisations de soins. L'instabilité des systèmes, saisie notamment par la théorie des systèmes sociaux développée par Niklas Luhmann (1984), permet d'aborder les organisations comme des constructions fortement improbables, aux dynamiques hétérarchiques formant un réseau de communication élargie. De ce fait, le changement n'est pas téléologique, c'est-à-dire avec des buts précis et précisés, et se fait selon des dynamiques risquées en introduisant de nouvelles pratiques de travail. Luhmann décrit ce mouvement comme l'« interpénétration » des systèmes, qui consiste en ce que les systèmes prêts à et capables d'ouvrir leur complexité créent d'autres systèmes dans leur environnement, lesquels intègrent de nouvelles informations, transformées en activités nouvelles et supplémentaires. Cette perspective auto-organisationnelle, ou autopoïétique, de Luhmann 
potentialise le sensemaking de K. Weick qui donne du sens à l'information entrante par le processus d' " adoption ».

Les points de vue épistémologiques des deux auteurs nous permettent d'aborder la notion de confiance fondamentale de façon générale dans la construction de la réalité. Pour Luhmann, elle est une dynamique de transformation graduelle en attente implicite de continuité focalisée sur des actions humaines. Sa mécanique se fonde sur la réduction de la complexité sociale. Il s'opère une transition de la confiance individuelle en l'autre à la confiance systémique. Nous pensons cette transition par le processus de l'« adoption » qui déclenchera l'engagement dans des actions au premier abord incertaines.

Au regard de cette hybridation conceptuelle, nous pensons que la communication construit l'organisation certes, mais selon des conditions spécifiques propices à l'émergence de nouvelles formes d'échanges. Une organisation de santé, système de normes héritées, qui installe un nouveau dispositif de normes, ajoute nécessairement un système dans le système, en créant les conditions d'informations supplémentaires qui verront leur assimilation pour autant qu'elles soient adoptées/adaptées et qu'elles modèrent les risques possibles, susceptibles de déséquilibre.

La situation de gestion du risque dans les établissements de santé qui installe le nouveau dispositif de vigilance « sur-anticipatif » du risque met à l'épreuve les coopérations, les liens et "l'acte de disposer " (Belin in Charlier et Peeters, 1999:251) des autres, des lieux, des techniques, de se les approprier pour s'y engager aussi en acte et en sens (F. Bernard, 2004). Mais l'enjeu de la communication dans l'évolution des organisations de santé reste dépendant du contexte de risques.

\section{Un contexte des risques en tension}

Le terme de risque s'inscrit dans le couplage anthropogénèse-anthropotechnique (Stiegler, 1996; Sloterdijk, 2000) qui a toujours marqué les progrès techniques et touché les domaines consubstantiels des organisations sociales humaines (risques industriels, économiques, sociaux, environnementaux). Les heurs et vertus des usages de la technique ont inauguré le débat éthique, qui se poursuit, sur le contrôle des effets à long terme des progrès scientifiques. En effet, le discours sur le développement technique n'est plus neutre dans ses conséquences sur la nature : «les conséquences néfastes dépassant parfois de loin le but recherché » (Jonas, 1997 : 232).

La mesure de la contingence et de l'aléa attachés à la notion de risque demeure la principale préoccupation des organisations. Elle est associée à la prédiction comme horizon problématique - en référence aux notions de big data, normes, prédiction, maîtrise.... A l'anticipation et à la maîtrise des catastrophes naturelles d'une part et aux conséquences néfastes du déploiement technologique d'autre part, les sociétés répondent par une gestion 
des risques fondée sur une anticipation qui garantirait une maîtrise totale de notre sécurité. La prévention des risques s'organise, entre autres, autour de dispositifs d'information spécifiques, de nouvelles formes de prédiction (en particulier liées à la croissance des empiries numériques et à l'algorithmique) et d'espaces collaboratifs plus bottom-up que par le passé.

Plusieurs motifs président au fait que la santé soit toujours convoquée en arrière-plan de la notion de risque : ils sont humains (question de vie et de mort), éthiques (la santé est un "bien »), économiques (la santé a un coût). Et cela couvre un spectre assez vaste, entre des "scénarii-catastrophe » qui engendrent une politique de la peur et donc un principe de précaution extrême, et une progression des innovations technoscientifiques susceptibles d'améliorer nos conditions de vie.

Le risque a infiltré la société, accompagnant les meilleures et les pires transformations de notre environnement. Dans ce contexte, la circulation de l'information s'accompagne de plus en plus d'une prolifération des procédures, normes, évaluations dans les organisations de soins, prolifération qui modifie structurellement les pratiques professionnelles et entraîne le développement de nouvelles formes organisationnelles. La notion de risque donne un jour singulier aux processus de communication, en tension entre l'application stricte des prescriptions et la liberté de les dépasser, de les anticiper comme c'est le cas dans la prise en compte du near-miss.

\section{Risque et organisations de soins}

L'encadrement des risques pour les organisations de soins se complique dans un contexte de pression économique, marqué par une contrainte d'efficience sans cesse durcie. La question du coût est primordiale et les risques quantifiés, évalués sont soumis à variations d'opinion et impliquent des actions politiques parfois difficiles à cerner, à justifier, à mettre en place. Or, suivant un principe de cyndinique ${ }^{4}$ (Jousse, 2009), certains risques mineurs méritent aussi l'élaboration d'une méthode de prévention et ce, contre des principes d'efficience imposés par des économies politiques spécifiques de l'État.

Dans les organisations de santé, la gestion de la qualité des soins conjuguée à l'efficience intègre la prise en compte de tous risques majeurs (plans blancs) et mineurs (infections nosocomiales par exemple) jusqu'au risque encore non identifié. Un nouveau mode de communication systémique et structurant s'institue, fondé sur le paradigme de l'anticipation généralisée. Les établissements médicaux, depuis la loi HPST de 2009, doivent se référer aux normes de qualité pour « la gestion des risques associés aux soins » et veiller à la sécurité des patients hospitalisés.

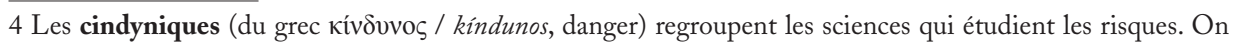
les appelle aussi « sciences du danger ». Elles s'intéressent plus particulièrement aux risques industriels et plus spécifiquement aux risques majeurs. Ce néologisme a été introduit lors d'un colloque tenu à la Sorbonne en 1987. Pour éviter une confusion entre « danger » et « risque »; en 2004, dans Le Risque, cet inconnu, Georges Jousse a proposé le terme « riscologie » pour l'étude générale et scientifique des risques. 


\section{$\mathrm{C} \& O \mathrm{n}^{\circ} 45$}

Une étude d'Ellenberg réalisée au moment de la mise en place de la procédure d'accréditation d'un centre hospitalier parisien, a permis d'établir une cartographie des responsabilités de la sécurité sanitaire dans l'hôpital pour proposer des «pistes managériales » (Ellengerg, 2003 : 63). De façon claire, il identifie deux types de structures qui prennent en charge la sécurité sanitaire : l'une préventive qui agit en amont pour tous les risques techniques, interne à l'institution, l'autre liée aux « vigilances » relatives aux " produits frontières " qui arrivent de l'extérieur (matériels biomédicaux, produits sanguins, médicaments...). Des deux méthodes, réactive et proactive, que décrit Ellenberg, celle proactive est la moins développée. " Finalement, les risques hospitaliers réglementés sont pris en charge conformément à la loi. » (Idem : 64) et il constate que chacun travaille de façon cloisonnée. « Peutêtre serait-il pertinent de créer un lieu d'échange entre les différentes unités d'un même hôpital (ou d'un autre) pour qu'ils s'informent respectivement de leur façon de travailler, car les approches face aux risques sont différentes " (Ibidem). Il est intéressant de constater qu'Ellenberg appréhende le risque à l'hôpital dans toutes ses dimensions médicales, juridiques et assurantielles pour que de la prévention à la protection, les patients (re)trouvent la confiance en l'établissement où ils sont hospitalisés. La charge est laissée au management de fédérer les équipes autour des risques et de les faire travailler sur le retour d'expérience (cf. le risk management) et la vérification de l'application des normes très contraignantes, la confiance des patients restant l'enjeu pour des établissements sous fortes contraintes. La nature de cette confiance est complexe, instable, soumise à tensions contradictoires.

S'affranchir de la gestion prescriptive et procédurale des instruments de l'État (Lascoumes, Le Galès, 2005), faciliter le repérage du risque d'événement non souhaité (ENS) et favoriser la prédiction d'une manière générale se concentre dans l'analyse des near-misses (McKinnon, Ron, 2012). Approche proactive, le processus communicationnel autour du near-miss sollicite le changement organisationnel, centré sur la prévention, et tend à privilégier les actions conjuguées de tous les salariés, plus motivés par la confiance qui leur est accordée et qu'ils accordent au système organisationnel.

\section{Étude du near-miss : une sur-anticipation du risque}

Le contexte général de risque et de sécurité sanitaire dans les établissements de santé ${ }^{5}$ combiné à la culture de l'efficience, a généré une veille accrue des événements indésirables associés aux soins (décret du 12/10/2010). La décision d'installer un nouveau dispositif de vigilance n'aurait rien eu d'extraordinaire car il est très proche du suivi habituel des événements indésirables, si deux points originaux n'étaient venus s'y associer :

5 Sécurité sanitaire dans les établissements de santé. Réglementation applicable. DHOS. Ministère de l'Emploi et de la Solidarité. www.sante.gouv.fr/htm/dossiers/ secu_san3/secusan3.pdf 
1. une démarche managériale originale, entre contrainte et liberté, qui d'une part ajoute aux procédures de gestion des risques déjà pesantes, la systématisation de la déclaration de near-miss, et d'autre part accepte toutes sortes de descriptions du " presqu'accident ", qu'elles paraissent anodines ou confuses, inutiles ou fantaisistes à son auteur,

2. une ouverture d'un espace-temps de discussions en commission vigilance à propos du signalement d'un near-miss : l'auteur de la fiche est invité à commenter et participer à l'élaboration de sa solution avec la volonté d'aplanir l'impact hiérarchique qui pourrait bloquer certaines informations ${ }^{6}$.

$\mathrm{Au}$ plan méthodologique, selon une approche compréhensive, nous avons assisté en tant qu'observateur-participant, à la mise en place de ce qui deviendra un dispositif consacré aux near-miss, considérés dans cet établissement comme la pierre angulaire de la gestion des risques (cf. Infra, la pyramide de Bird). Suivant une posture abductive, une enquête et l'écoute des acteurs concernés (patients et professionnels), nous présentons de façon non académique, itérative, l'ensemble complexe info-communicationnel de la procédure d'analyse de near-miss.

\section{Définition du near-miss}

Létablissement de santé privé ${ }^{7}$ qui installe la procédure du near-miss de notre étude, est réputé pour ses performances en matière de qualité globale (soins, sécurité) et pour ses démarches innovantes.

Cette procédure du near-miss s'apparente à celle des événements indésirables (EI) qui peuvent être graves (EIG). L'EI s'intègre dans l'attention portée à la sécurité du patient ( $\mathrm{C}$ f. la réglementation de la Haute Autorité de Santé, HAS de 20118). "Il peut aussi être sans conséquence s'il est détecté et récupéré à temps. On parle alors de "near-miss » ou d'événement porteur de risque (EPR) (au Québec on parle d' "échappée belle ») dont l'analyse est aussi riche d'enseignements qu'un EIG » (HAS, 2012, Ibidem). Dans le registre nearmiss, la surveillance des " quasi-accidents " permet des actions correctives, prévient la récidive et évite l'accident. En effet, un near-miss est un événement

6 Les relations hiérarchiques sont à entendre ici dans leur nature administrative, mais aussi symbolique entre soignants relativement au pouvoir encore très prégnant des médecins. Ceux-ci sont impliqués dans la déclaration d'événements indésirables depuis longtemps.

7 L'établissement assure la prise en charge des patients en services de Médecine, Chirurgie et de GynécoObstétrique. « L'Etablissement, qui a reçu la visite de certification V2010 des experts de la HAS en avril 2013, est engagé depuis de longues années dans une démarche qualité et sécurité des soins plaçant la satisfaction des patients au cœur des priorités ». Ce message en présentation du site n'est pas uniquement déclaratif puisque l'établissement outre les certifications successives "sans réserve " de la Haute Autorité de Santé, instance évaluative, culmine en tête dans les classements médiatisés dans la catégorie des établissements entre 100 et 300 lits.

8 « Les systèmes de signalement des événements indésirables en médecine se répartissent en trois grandes classes. La classe I regroupe les systèmes passifs reposant sur la déclaration des acteurs médicaux, la classe II les systèmes passifs reposant sur la déclaration des patients et la classe III rassemble les systèmes actifs fondés sur l'analyse de dossiers ou de traces électroniques » http://www.has-sante.fr/portail/jcms/c_1098577/ $\mathrm{fr} /$ declaration-des-evenements-indesirables 


\section{$\mathrm{C} \& O \mathrm{n}^{\circ} 45$}

imprévu qui n'a pas entraîné de blessure, de maladie ou de dommage, mais qui avait le potentiel pour le faire. Seule une rupture considérée comme une chance, dans la chaîne des événements, a empêché l'accident. Dans la vision probabiliste de survenance des accidents, la détection des presqu'accidents aurait une action significative sur la baisse des accidents graves.

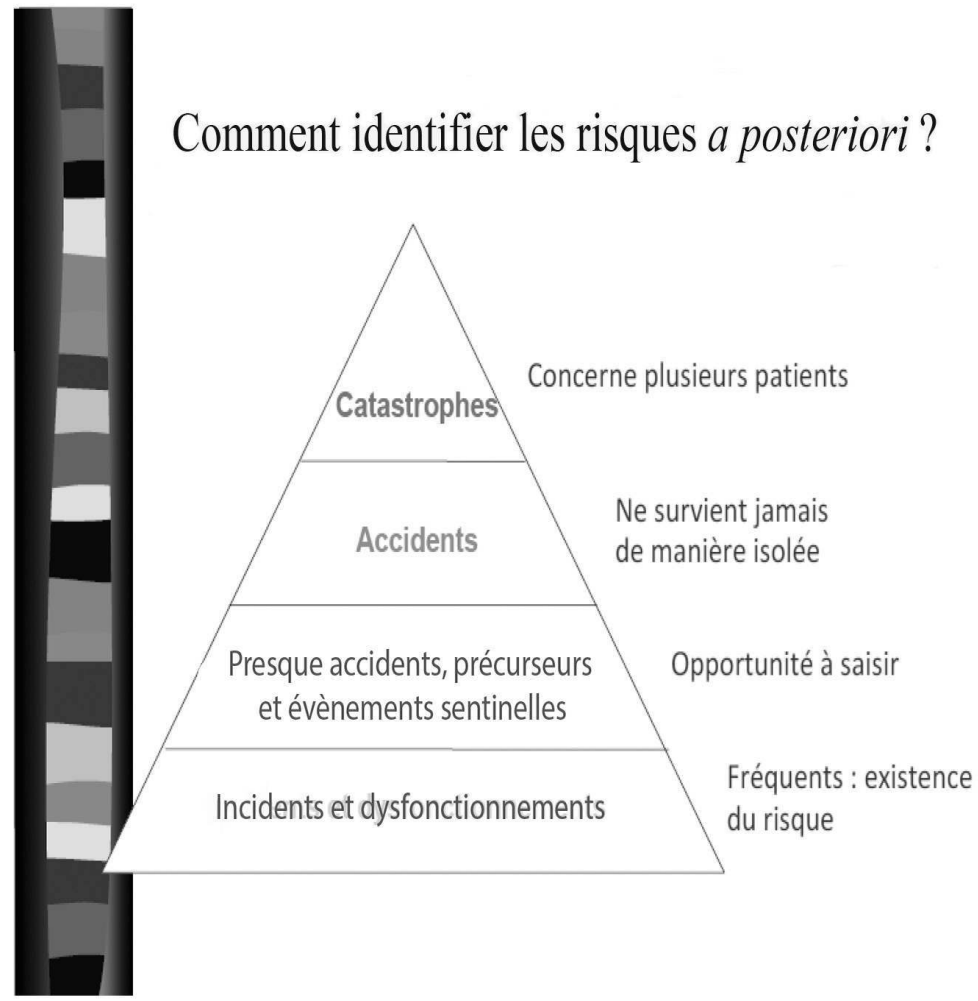

Pyramide revue par la cellule qualité de l'établissement inspirée des travaux de Heinrich HW (1931). Industrial accident prevention: a scientific approach, Montreal, McGraw-Hill

Inspirée de la Pyramide de Bird (1969), ce classement indique que si l'organisation parvient à réduire le nombre d'incidents et de presqu'accidents, le nombre d'accidents au sommet sera diminué. La notion de near-miss ou " presque accident » n'est pas courante encore dans le contexte de la gestion des risques en santé. En effet, essentiellement abordée par la gestion, elle passe par la comptabilité des accidents et le relevé taxinomique comme référence de ce qu'il faut éviter. Le near-miss est considéré comme un événement porteur de risque (EPR) n'ayant pas engendré de conséquence grave pour le patient, dont l'analyse méthodique favorisera la compréhension des causes de survenue, mais aussi celle des modalités de la récupération des informations 
ayant permis à temps sa détection et son traitement. Si l'intérêt de ces relevés de near-miss paraît évident, on comprend bien que les personnels puissent être préoccupés quant à l'ajout d'une nouvelle tâche.

\section{Déclaration du near-miss}

Cet établissement de soins invente une nouvelle organisation de vigilance dans laquelle tous les professionnels soignants et administratifs sont invités à s'impliquer à la suite d'une formation aux " presqu'accidents ", menée par la responsable qualité de l'établissement. Chaque recruté reçoit cette formation. La démarche est simple : tous les personnels, quelle que soit leur position hiérarchique, peuvent assurer un signalement au moyen d'une fiche d'événement indésirable (FEI), informatisée et gérée par le logiciel Blue médi santé 9 . Tous les postes informatiques en sont équipés et fonctionnent en réseau. Les personnels utilisent le logiciel pour le dépôt de leur déclaration.

Ce protocole de signalement est signé par l'auteur qui est invité, lors du traitement des fiches en commission, à s'exprimer sur les solutions envisagées. La législation préconise une efficacité accrue des FEI en déculpabilisant celui qui rédige la fiche, car il peut être perçu comme un dénonciateur lorsqu'il implique nominativement un collègue. La politique est ici clairement dans l'écoute neutre et bienveillante de tout auteur de FEI et/ou de near-miss, et la commission vigilance est un lieu d'échange et d'étude du near-miss.

Un personnel agent des services hospitaliers (ASH) par exemple, écrit sur la FEI « un patient que je brancardais sur un fauteuil roulant, du scanner jusqu'à sa chambre, s'est mis à vomir du sang dans le couloir. Il n'y a pas de téléphone dans le couloir et aucun soignant n'est avec moi ». Au moment du traitement de la fiche, l'ASH prend la parole, le radiologue participe aussi pour comprendre et d'autres personnels interviennent pour faire une " proposition d'actions d'amélioration " : installer des téléphones à intervalles rapprochés dans les couloirs, équiper les personnels de téléphones portables, équiper les murs de boutons d'alarme....

88 personnels sur les 232 de l'établissement ont été interviewés. Dans un premier temps, ils disent avoir reçu l'information d'une nouvelle procédure de vigilance concernant des presqu'accidents, comme une « charge supplémentaire inutile ", imposée par une direction " parano ». Dans un deuxième temps, $67 \%$ de ceux qui ont participé au protocole near-miss et à la commission vigilance, indiquent que la qualité de l'attention reçue et la concrétisation de solutions lors des débats :

- ont modifié positivement l'estime de soi, des autres, de l'établissement grâce à sa contribution à l'amélioration de la qualité,

- ont accru la vigilance sur la sécurité des patients,

- ont facilité les échanges interprofessionnels. 


\section{$\mathrm{C} \& O \mathrm{n}^{\circ} 45$}

Tous les personnels qui s'associent au dispositif, finissent par être pris dans une dynamique communicationnelle hors hiérarchie, dont l'expérience répétée génère et installe la confiance. En effet, le dispositif permet de réduire la complexité d'un environnement a priori non maîtrisé d'où peut sortir un danger pour la sécurité des patients. Les personnels marquent leur engagement par la vigilance qui accompagne leur pratique. En effet, $906 \mathrm{FIE}$ ont été réalisées en 2013 dans cet établissement de 300 lits. L'entrecroisement des processus communicationnels et de confiance reconfigure l'organisation dans ce sous-système d'une gestion particulière des risques. La contrainte imposée par la gouvernance d'une sur-vigilance est assimilée, absorbée par le dispositif near-miss.

\section{Logiques organisationnelles entre communication et confiance}

Des artefacts cognitifs, matériels, ses actants et leur coordination reconfigurent communicationnellement l'organisation qui prend une forme originale, car appropriée conjointement par les salariés et la direction. Ce processus d'«adoption » décrit parWeick, dans le système hétérarchique assumé par une logique managériale pro-active que préconisait Ellenberg (2003, op. cit.), installe les conditions organisantes de la communication attachée au dispositif du near-miss. En effet, les médecins sont dans le dispositif des near-miss, participent à la création au même titre que l'ensemble des salariés ; ils ne jouissent pas de prérogative particulière, ni réelle ni symbolique qui serait due à leur statut ${ }^{10}$.

Il est possible de penser que ce dispositif deviendra une norme légitimée par les professionnels car il est fondé sur des raisons collectives et institutionnelles " résultant d'un processus de délibération inclusif et équitable " comme le dit Habermas (in Blondiaux et Sintomer, 2002 : 19). Dans ce type de dispositif effectivement, tous les salariés peuvent participer et sont amenés à coopérer librement. C'est bien cet espace délibératif où s'ouvre un espace de créativité pour trouver des solutions adaptées aux locaux, aux personnels, aux activités pour la sécurité et le confort des patients. Le nouveau système de near-miss se crée dans celui plus général de gestion des risques. Le principe d'interdépendance des systèmes permet l'émergence d'un système dans le système, comme le décrit Luhmann.

10 Il faut savoir que l'un des points essentiels du dispositif national d'accréditation des médecins est l'analyse des événements indésirables porteurs de risques (EPR) déclarés par les médecins inscrits dans cette procédure. Ce sont les événements indésirables associés aux soins qui n'ont pas causé de dommage grave au patient. Audelà de la recherche des causes immédiates et des facteurs favorisants, l'un des axes de travail porte sur les modalités de la récupération avec ses temps de détection (qui, quand, comment), de diagnostic (qui, quand, comment) et d'action (qui, quand, comment). L'enjeu est majeur : identifier au sein de chaque spécialité des situations à risques méconnues, étudier grâce à une base de données (base REX) des " paniers " d'EPR semblables pour tenter d'identifier des causes communes. L'objectif de cette procédure est d'élaborer des recommandations professionnelles en conséquence, rédigées sous la forme de barrières de sécurité. Le rapport d'étude INERIS (2004 et 2008) portant sur les « enquêtes sur la gestion des presque accidents et systèmes de retour d'expérience " précise que tous les acteurs doivent y être associés. 
Le dispositif des near-misses montre comment des processus communicationnels organisants, originaux et créatifs modifient conséquemment les formes organisationnelles de santé ordinairement enserrées dans des normes administratives. Ce système de gestion des risques s'auto-génère au fur et à mesure de la confrontation aux difficultés en train d'apparaître. La communication participe à cette réélaboration/reconstitution organisationnelle autour d'une norme émergente (celle de l'ultra-prévention du near-miss dans l'arsenal normatif de la gestion des risques) dans la logique de l'interpénétration des systèmes où advient le sensemaking weickien et qui, en d'autres termes, rejoint la notion d'engagement « en acte et en sens » de F. Bernard (op. cit.).

La confiance selon Luhmann "se crée par une extrapolation à partir de l'information disponible; elle est, comme Simmel l'a noté, un mélange de savoir et de non-savoir. » (Luhmann, 1968-2006 : 28). Elle participe au processus d'engagement des personnels dans une vigilance accrue et se diffuse au collectif ainsi quaux patients. "La confiance ouvre, au moyen d'une réduction de la complexité, des possibilités d'action qui, sans elle, demeureraient improbables ou encore peu attrayantes, qui ne se réaliseraient donc pas " (Ibidem : 27). La confiance individuelle en la direction de l'établissement est transférée au système organisationnel.

\section{Conclusion : La confiance dans l'action}

Selon la perspective luhmannienne des systèmes sociaux complexes que nous croisons avec la théorie de la construction du sens, cette dynamique préventive s'intègre dans le processus de changement des formes organisationnelles par la réduction de la complexité, réduction générative de confiance. En effet, cette réduction s'opère dans les organisations de santé par le focus que les professionnels sont capables de faire notamment sur un " non-événement ", qu'ils acceptent comme indiciel d'un événement potentiellement dangereux. La consignation écrite et la prise en compte de cet écrit débattu dans des espaces qui satisfont un "impératif délibératif » (Sintomer, Blondiaux, 2002 : 17) dans un mouvement bottom-up, laissent la possibilité d'investissement et de responsabilisation des professionnels dans la prise en compte de risques éventuels pour les patients et les salariés.

Les effets de ces communications organisantes, langagières et non langagières, concourent à l'élaboration d'interactions fondées sur la confiance organisationnelle, et modifient les relations de pouvoir. La gestion des risques ainsi fondée sur des logiques professionnelles créatrices induit un changement non seulement dans les pratiques, mais aussi dans la signification après coup des décisions et des actions, résultantes d'une intelligence collective, distribuée et située. Dans les organisations de santé, systèmes auto-organisés, les normes techniques héritées (Le Moënne, 2004) des instruments de l'État ne peuvent être modifiées, réadaptées, prolongées que par l'engagement de tous les acteurs de terrain. La prévention des risques selon le dispositif de vigilance aux near- 
misses est, au-delà de l'application des normes imposées, une recomposition organisationnelle qui sopère par une nouvelle norme anthropologique émergente (Heylighen, 2011), susceptible d'enrayer certains risques dans et par la confiance communicationnelle diffusée à tous, professionnels et patients.

\section{BIBLIOGRAPHIE}

BATESON Gregory, Steps to an ecology of mind, San Francisco, Chandler Press, 1987, $421 \mathrm{p}$.

BECK Ulrick, La société du risque, sur la voie de la modernité, Champs essais, Paris, Flammarion, 1986.

BELIN Emmanuel, « De la bienveillance dispositive », in Charlier, Philippe., Peeters, Hugues., Hermès 25, 1999, p. 245-259.

BERNARD Françoise, ROBERT-VINCENT Joule, « Lien, sens et action : vers une communication engageante ", Communication et organisation [En ligne], 24 | 2004, mis en ligne le $1^{\text {er }}$ avril 2012, consulté le 03 avril 2014. URL : http://communicationorganisation. revues.org/2918

BOUILLON Jean-Luc, BOURDIN Sylvie, LONEUX Catherine, " De la communication organisationnelle aux « approches communicationnelles " des organisations : glissement paradigmatique et migrations conceptuelles », Communication et organisation [En ligne], 31, 2007, mis en ligne le 1er juillet 2010, consulté le 24 octobre 2012. URL : http://communicationorganisation.revues.org/90

ELLENBERG Eytan, «Le management des risques à l'hôpital », adsp, n 45, décembre 2003, p. $62-66$.

FHP, GDR, infections associées aux soins, la gestion des risques en établissement de santé ? Publié le 21 décembre 2011.

HEYLIGHEN Francis, «Self-organization in Communicating Groups: the emergence of coordination, shared references and collective intelligence», in Language and Complexity, Barcelona University Press, 2011.

JONAS Hans, "La technique moderne comme sujet de réflexion éthique ", in $L a$ responsabilité. Questions philosophiques, Marc Neuberg (ed), 1997.

JOUSSE Georges, Traité de riscologie - La science du risque, Imestra Éditions, 2009.

LASCOUMES Pierre, LE GALÈS, Patrick, Gouverner par les instruments, Coll. Académique, Paris, Presses de Sciences Po, 2005.

LE COZE Jean-Christophe, LIM Samantha, DECHY Nicolas, "Enquêtes sur la gestion des presque accidents et systèmes de retour d'expérience ", Rapport d'étude 31/7/2008, INERIS-DRA-08-95321-10619A

LE MOËNNE Christian, La communication organisationnelle à l'heure de la dislocation spatio-temporelle des entreprises, Toulouse, Sciences de la Société, $n^{\circ}$ 62, 2004.

LUHMAN Niklas, Systèmes sociaux: Esquisse d'une théorie générale, Presses de l'Université Laval, 1984, trad. 2010.

MCKINNON Ron C., Safety Management: Near-miss Identification, Recognition, and Investigation, CRC Press, 2012. 
SINTOMER Yves., BLONDIAUX L., « L'impératif délibératif », Politix, Année 2002, Volume 15, n 57, p. 17-35.

Un avenir à haut risque, L'Observateur de l'OCDE N ${ }^{\circ} 235$, décembre 2002. http:// www.observateurocde.org/news/archivestory.php/aid/752/Risques_futurs.html\#sthash. JpCCRhFB.dpuf

WEICK Karl E, Sensemaking in Organizations, Sage publications, 1995, 237 p.

WINKIN Yves, La nouvelle communication, Paris Seuil, 2000, 182 p.

Résumé : Dans les organisations de santé, la prise en compte des risques pour les patients et les professionnels, suppose d'un côté une gestion procédurale de leurs conséquences suivant une démarche réactive, et de l'autre l'anticipation de leurs effets selon une autre pro-active. Encadrées par une réglementation de plus en plus pointue, elles s'intègrent dans la démarche qualité des établissements de soins. Au-delà de la gestion applicative, les recherches en communication organisationnelle nous permettent d'affiner la compréhension de l'implication des acteurs dans l'anticipation des risques à travers l'étude du near-miss ou « presqu'accident ». Le traitement du near-miss fonde et installe la confiance à la base d'une communication intégrative et constitutive du système organisationnel. Cette recherche montre une évolution relative aux normes qui régissent les risques dans les organisations de santé et les reflexions théoriques qu'elle soulève.

Mots-clés : communication organisationnelle, risques, confiance, changements, nearmiss.

Abstract: In healthcare organizations, taking into account risks to patients and professionals, supposes a procedural management of their result in a reactive approach and anticipate their effects in a pro-active one. Regulations framed by increasingly sharp, these steps are part of the quality of residential care approach. Beyond application management, researches in organizational communication allow us to refine our understanding of the involvement of stakeholders in anticipation of risks through the study of near-miss or almost accident. Treatment of the nearmiss built and installs confidence in the basis of an integrative and constitutive organizational communication system. This research shows an evolution on standards governing risk in healthcare organizations and theoretical reflections it raises.

Keywords: Organizational Communication, Risk, Confidence, Changes, Near-miss. 
